\title{
¿En qué Ciudadanía Creen los Jóvenes? Creencias, Aspiraciones de Ciudadanía y Motivaciones Para la Participación Sociopolítica
}

\section{What Type of Citizenship Do Youth Believe in? Beliefs, Aspirations, and Motivations for Sociopolitical Participation}

\author{
M. Loreto Martínez, Carmen Silva y Ana C. Hernández \\ Pontificia Universidad Católica de Chile
}

\begin{abstract}
En esta era global los jóvenes se han distanciado de formas tradicionales de ejercer su derecho político (e.g., voto) y han mostrado nuevas formas de participación sociopolítica cuyo sustrato de creencias y motivaciones ha sido poco estudiado. Se indagan las creencias y aspiraciones de ciudadanía en una muestra de 24 jóvenes chilenos, de ambos sexos y diversa condición socioeconómica, todos con historia de participación en organizaciones de voluntariado social, políticas, étnico-políticas, ecológicas y estudiantiles. Se utilizó una metodología cualitativa, con entrevistas individuales semiestructuradas y grupos focales. Los resultados indican que la motivación central de los jóvenes es cambiar la falta de validación de las personas en el sistema sociopolítico actual, debido a la vulneración de derechos, inequidad social y falta de voz y poder de los ciudadanos. Los jóvenes adscriben a una ciudadanía activa que valora la responsabilidad de informarse y la deliberación de los ciudadanos. A la base de sus creencias y aspiraciones de ciudadanía emergen los valores de fraternidad, dignidad y equidad.
\end{abstract}

Palabras clave: ciudadanía, jóvenes, creencias, participación social

\begin{abstract}
In current global age youth have drifted apart from traditional forms of exercising political rights (e.g., voting) and have engaged in new forms of social and political participation. The beliefs and aspirations of such forms of citizenship and the motivations underlying new forms of participation have not been the focus of research. This study explores the beliefs and aspirations of citizenship in a sample of 24 Chilean youth, males and females of diverse socioeconomic condition. Participants had a history of participation in political, ethno-political, and ecological organizations. A qualitative methodology with in-depth semi-structured individual interviews and focus groups was used. Findings indicate that youth's central motivation is to change the lack of validation of people within the current sociopolitical system, due to vulnerability of rights, social disparities, and lack of voice and power of citizens. Participants endorse a notion of citizenship that values citizens' responsibility to be informed and to deliberate. Values of fraternity, dignity, and equity underlie youth's beliefs and aspirations of citizenship.
\end{abstract}

Keywords: citizenship, youth, beliefs, social participation

Además de un conjunto de derechos y prerrogativas, la ciudadanía comprende un conjunto de prácticas jurídicas, políticas, económicas y culturales que definen la membresía social y que, como consecuencia, moldean el flujo de recursos a personas y grupos sociales (Turner, 1993).

El significado más común de ciudadanía es ser miembro de una comunidad. Ser ciudadano conlleva pertenecer a cierta co-

M. Loreto Martínez Guzmán, Carmen L. Silva Dreyer y Ana Cecilia Hernández Sandoval, Escuela de Psicología, Pontificia Universidad Católica de Chile, Santiago, Chile.

Este trabajo fue realizado como parte del Proyecto FONDECYT N 1085231 Desarrollo Cívico en Jóvenes Chilenos. Parte del estudio fue financiado por la Vicerrectoría Adjunta de Investigación y Doctorado de la Pontificia Universidad Católica de Chile, a través del Proyecto Límite 2007/08.

La correspondencia relativa a este artículo debe ser dirigida a M. Loreto Martínez Guzmán, Escuela de Psicología, Pontificia Universidad Católica de Chile, Avda. Vicuña Mackenna 4860, Macul, Santiago, Chile. E-mail: mlmartig@uc.cl 
munidad política, reconocer y ejercer los derechos civiles, políticos y sociales y cumplir con deberes y responsabilidades en la conducción de la vida común (Martínez, 1999). Sin embargo, existen distintos enfoques para el estudio de la ciudadanía (e.g., republicanismo cívico, democracia participativa, liberalismo) como también concepciones disímiles en los diferentes actores de la sociedad (e.g., jóvenes, minorías).

En Chile la visión oficial de ciudadanía sustentada en la Constitución coexiste con un llamado a la participación ciudadana ampliamente proclamada por autoridades políticas, dentro de un modelo económico y democrático liberal. En este, el rol ciudadano se ejerce principalmente a través del sufragio y la participación en instancias micro y meso sociales (e.g., juntas de vecinos, organizaciones sociales a nivel local), en el marco de una transferencia de poder desde el Estado al mercado (Silva, 2009). Sin embargo, las instituciones políticas y las autoridades que las representan no son los únicos actores en un escenario democrático.

Los jóvenes son agentes clave en el proceso de renovación de la sociedad, pues constituyen la generación que se hará cargo de la conducción del país. En Chile estas generaciones han sido socializadas en las prerrogativas de la democracia y, comparativamente con generaciones anteriores, influidas menos directamente por el impacto sociopolítico del régimen autocrático que restringió la participación y derechos ciudadanos durante 1973-1990. Evolutivamente, los jóvenes están en proceso de configuración de su identidad, es decir, de formarse su propia visión sobre la sociedad en cuanto a asuntos políticos, económicos y sociales y de adoptar un sistema de valores o una ideología que guíe su accionar (Erikson, 1968).

Algunos estudios (Instituto Nacional de la Juventud [INJUV], 2009; Programa de las Naciones Unidas para el Desarrollo [PNUD], 2004) sugieren que la concepción de ciudadanía de los jóvenes no coincide con la oficial y que sus expectativas de participación social no se enmarcan en los cánones tradicionales de participación política. Uno de estos signos es el masivo desinterés por la inscripción en los registros electorales (INJUV, 2009) y la desafección de muchos jóvenes con la política convencional. Alternativamente, la masiva movilización de los estudiantes secundarios (Domedel \& Peña y Lillo, 2008) y universitarios en 2006 en pro de una mejor calidad de la educación, el interés por participar en proyectos sociales y/o voluntariados y en una variedad de grupos liderados por jóvenes (e.g., colectivos, culturales, sociales), virtuales o presenciales (INJUV, 2007), demuestran su interés en asuntos públicos.

Las observaciones anteriores sugieren un desencuentro de expectativas entre la manera en que la institucionalidad cree que los jóvenes debieran comprometerse y las creencias, aspiraciones y motivaciones cívicas propias de los jóvenes. Si efectivamente los jóvenes no comparten esta visión, ¿cuál es su creencia de ciudadanía? ¿A qué tipo de ciudadanía aspiran? ¿Qué motivaciones subyacen a su participación sociopolítica?

Estos tópicos han sido poco investigados en Chile. Algunos estudios han caracterizado la participación juvenil (INJUV, 2004, 2007), documentando sus efectos para el desarrollo de competencias (Silva \& Martínez, 2007; Velásquez \& Martínez, 2004; Youniss et al., 2002) y las condiciones que favorecen el desarrollo sociopolítico en los jóvenes (Ginwright \& James, 2002; Watts \& Flanagan, 2007). Sin embargo, las creencias y aspiraciones de ciudadanía que subyacen a las formas de participación social elegidas por los jóvenes no han recibido la misma atención y constituyen el foco de este estudio.

\section{La Ciudadanía en los Jóvenes}

La ciudadanía como concepto y práctica de convivencia social es histórica y cultural (Bárcena, 1997), es decir, evoluciona desde determinados enfoques teóricos y valóricos en respuesta a las características del contexto político, social y económico. Diferentes enfoques del concepto de ciudadanía, como el liberalismo, comunitarismo, republicanismo cívico, democracia participativa, teorías postmodernas (Janoski \& Gran, 2002) y ciudadanía crítica (Knight \& Harnish, 2006) acentúan, ya sea los derechos individuales, metas comunitarias, participación o derechos particulares, como la pertenencia 
e identidad de grupos excluidos.

La realidad actual de las naciones inmersas en un sistema global que escapa a la regulación del Estado, de actores sociales y de sujetos individuales, exige repensar el término (Martínez, 1999). La juventud es la generación influida más plenamente por esta época, por lo que resulta relevante preguntarse por sus creencias y aspiraciones en relación a la ciudadanía. Se entiende por creencias tanto sus definiciones y apreciaciones de la ciudadanía, como los significados que asocian a esta práctica, y por aspiraciones, su ideal de ciudadanía asociado asimismo a sus definiciones de la misma.

Diversos autores (Flanagan, 2003; Youniss et al., 2002) consignan menor participación juvenil en política convencional a nivel mundial y mayor asociatividad en torno a voluntariados sociales y acciones locales y puntuales. Garcés (2010) describe un cambio desde la política tradicional a los colectivos, agrupados en torno a derechos de categorías sociales, cultura, identidades y derechos humanos. Sandoval y Hatibovic (2010) describen una difícil relación entre la política convencional y los jóvenes. Las formas de participación social preferentes son la actividad comunitaria y campañas virtuales, y las menos preferidas, la campaña política y la política partidaria (INJUV, 2009). Los hallazgos anteriores indican la importancia de conocer, desde la perspectiva de los jóvenes, las creencias, aspiraciones de ciudadanía y motivaciones para involucrarse en asuntos de la sociedad.

\section{Método}

El estudio se realizó en dos etapas. En la primera, se indagaron las concepciones de ciudadanía y las motivaciones de participación social o política a través de entrevistas individuales en profundidad. Los hallazgos de las entrevistas sirvieron de base para la segunda etapa, en la que se replicó la indagación en una muestra más amplia de jóvenes, examinando también aspectos más colectivos de las experiencias de participación social y política a través de grupos focales.

\section{Participantes}

Muestra para entrevistas individuales. Fue seleccionada por medio de contactos con diversas organizaciones sociales, de voluntariado y políticas que trabajan con jóvenes en la Región Metropolitana de Santiago de Chile. Para tener una representación de creencias y motivaciones de jóvenes en diferentes etapas de vida, se seleccionaron tres jóvenes estudiantes de enseñanza media (edades entre 16 y 18 años) y tres jóvenes estudiantes universitarios de diferentes carreras (edades entre 21 y 24 años). La situación socioeconómica de los participantes era diversa, desde nivel socioeconómico (NSE) medio-bajo hasta medio-alto. Los participantes pertenecían a organizaciones cuyos objetivos se orientaban al voluntariado social (2), causas políticas (1), étnico-políticas (1), ecológicas (1) y organizaciones estudiantiles (1) que representaban una diversidad de contextos y de ideologías organizacionales. Los jóvenes entrevistados tenían una trayectoria de participación en sus respectivas organizaciones sociales y/o políticas de entre tres y siete años.

Muestra para grupos focales. Los participantes fueron 18 jóvenes de ambos sexos, de edades entre 16 y 25 años, estudiantes de enseñanza media y superior, de diferente NSE (desde medio-bajo hasta medio-alto) y todos con historia de activa participación social.

Se realizaron tres grupos focales. El primero $(n=5)$ estuvo compuesto por estudiantes de enseñanza media (16 a 18 años), residentes de una comuna de la zona sur de Santiago (NSE medio-bajo). Al momento de la realización del grupo focal, estos jóvenes estaban participando en organizaciones políticas (3) y estudiantiles (2) por períodos que fluctuaban entre 1 año 6 meses y 3 años 6 meses. Adicionalmente, su historia registraba participación en centros de alumnos, organizaciones de estudiantes, centros culturales y grupos ecológicos.

El segundo grupo focal $(n=5)$ estuvo compuesto por estudiantes universitarios (19 a 22 años), en su mayoría de NSE medio 
y medio-alto, residentes de diversas comunas de la Región Metropolitana. Estos jóvenes estaban participando en programas de voluntariado (3) y organizaciones políticas (2) por períodos que fluctuaban entre 1 año 3 meses y 4 años. Adicionalmente, registraban historia de participación en programas de voluntariado, centros de atención diurna, proyectos sociales, misiones pastorales y taller de ecología.

El tercer grupo focal $(n=8)$ estuvo compuesto por estudiantes universitarios (20 a 25 años), en su mayoría de NSE medio, residentes de diversas comunas de la Región Metropolitana. Estos jóvenes estaban participando en organizaciones políticas (partidos y colectivos) y estudiantiles (centros de alumnos y federación de estudiantes) por períodos que fluctuaban entre uno y seis años. Adicionalmente, registraban historia de participación en comunidades religiosas y voluntariados, la que, en algunos casos, se remontaba a varios años.

\section{Instrumentos}

Se utilizó una entrevista semiestructurada en formato individual y un grupo focal. Para cada instancia, se elaboraron guiones temáticos en base a las dimensiones identificadas en la literatura sobre desarrollo cívico en jóvenes, a saber, comportamental, intrapersonal, cognitiva y de socialización, como también formas de participación, motivaciones para la participación, visión y aspiraciones de ciudadanía, experiencias de socialización y el desarrollo de competencias cívicas. En el caso de los grupos focales, el guión también permitió a los participantes compartir opiniones. La información audiograbada de cada entrevista y grupo focal fue transcrita para su análisis.

\section{Procedimiento}

Los jóvenes fueron contactados a través de diversas instituciones y organizaciones sociales y ecologistas e invitados a participar voluntariamente en el estudio. Se explicaron los objetivos del estudio y se obtuvo consentimiento informado de los participantes. En el caso de menores de edad se solicitó consentimiento de los padres y asentimiento de los menores. El estudio fue aprobado por el comité de ética de la institución patrocinante.

Las entrevistas y los grupos focales fueron realizados por psicólogos, conducidos de acuerdo a los guiones de entrevista previamente elaborados, y tuvieron una duración de dos horas y media aproximadamente. Durante cada grupo focal, dos observadores entrenados registraron la interacción entre los participantes del grupo.

Los participantes fueron compensados con un bono de movilización y un refrigerio.

\section{Análisis de Datos}

La información obtenida a través de las entrevistas en profundidad y los grupos focales fue codificada de acuerdo a los lineamientos de la grounded theory (Strauss \& Corbin, 1990), enfoque que captura la fidelidad del fenómeno. La codificación se realizó en etapas sucesivas, descriptiva y relacional, y se postularon categorías que capturaran los contenidos de las respuestas de los participantes.

Análisis de entrevistas semiestructuradas. Se realizó una codificación abierta de las respuestas a las preguntas de cada entrevista, identificando y refinando categorías conceptuales que dieran cuenta de los contenidos. Se realizaron tres codificaciones abiertas independientes de cada entrevista $\mathrm{y}$, a través de un proceso de triangulación de investigadores, se analizaron las concordancias y discrepancias en la categorización de las respuestas. Finalmente, una cuarta codificación integró los acuerdos de las codificaciones independientes de cada entrevista, logrando la categorización y jerarquización definitiva de categorías. Las categorías resultantes de la codificación abierta se utilizaron como base para la descripción de los resultados. Con posterioridad a este análisis, se realizó la codificación axial, en la cual se establecieron relaciones entre las categorías definidas en la codificación abierta. Estas se organizaron en torno a un fenómeno central, a partir del cual se construyó un modelo comprensivo de las creencias y aspiraciones de ciudadanía de los participantes. 
Análisis de grupos focales. Se realizaron dos codificaciones independientes de las respuestas de los participantes y, por medio de triangulación, se analizaron las concordancias y discrepancias en la categorización de las respuestas entre codificadores independientes. Por medio de una tercera codificación se resolvió la categorización y jerarquización definitiva. Las categorías fueron postuladas en base a múltiples ejemplos de respuesta, lo que provee evidencia de la saturación de los contenidos.

A través de una triangulación de técnicas, se compararon las categorías que emergieron del análisis de las entrevistas con aquellas de los grupos focales. Se observó alta concordancia entre los resultados de ambos análisis, un indicador de la validez de los hallazgos. Las categorías fueron organizadas en torno a dos ejes temáticos, las motivaciones para la participación cívica y los valores a la base de sus aspiraciones como ciudadanos.

\section{Resultados}

Los resultados indican que las motivaciones de los jóvenes para la participación se sustentan en definiciones y aspiraciones de ciudadanía que entran en contradicción con sus experiencias como ciudadanos en la realidad. Los participantes perciben diferencias entre lo que aspiran y lo que observan en dos aspectos de la ciudadanía, la vulneración de derechos y la capacidad de influencia de los ciudadanos. Del análisis de sus críticas y sus propuestas para el cambio social emerge su compromiso con valores humanos que desean cristalizar en la ciudadanía.

\section{Motivaciones a la Base de la Participación: Creencias Sobre la Ciudadanía Actual}

Las motivaciones de participación se sustentan en una creencia de connotación crítica sobre el estado de la ciudadanía actual. En su análisis de la realidad los participantes perciben fallas en el sistema económico, social y político que desean cambiar. Los participantes muestran diferentes grados de elaboración en su comprensión de los problemas que identifican:

$Y$ ha visto, no con todos, él me cuenta que conoce personas que no tienen casa, que viven en una carpa en el sur en la octava región. Y que no tienen baño, hacen ahi en el campo. $Y$, ah, cuando (...) yo fui a la casa andaba buscando zapatos porque había conocido a la hija (...) una niñita chiquita como de tres años, no tenía zapatos. Su fuego fogón era afuera de la casa con lluvia. Y es terrible, terrible que pase porque esa gente no tiene un pedazo de tierra donde cultivar. Si tuviera un pedazo de tierra podría tener, tener para comer, podría tener para surgir, tener su casa. Pero no existe, eso no pasa en las tierras, las tienen unas (ciertas) personas para explotarlas, que después no sean no se puedan cultivar de nuevo, la gente queda botá (...). Las condiciones laborales igual no son buenas, son pésimas en muchos sectores, pésimas, inhumanas en muchos sectores, $y$ eso es lo que me, a mí me molesta. A mí me molesta que mi gente esté mal (...). Pero hay gente que puede dar mucho sacrificio y no tiene los medios como trabajar. Y eso es lo que me preocupa y yo creo que es el sistema imperante que nos tiene asi botados y hay que cambiarlo, definitivamente (E2, 189)

La motivación medular de estos jóvenes es cambiar la falta de validación o desconsideración de los derechos de las personas en el sistema político, debido a injusticias, inequidad y falta de poder y voz real de los ciudadanos. Los participantes aspiran a vivir en una cultura en la cual (a) las personas sean validadas ("cuentan e importan") en una vida comunitaria fraterna; (b) los derechos sociales sean respetados en un contexto de equidad (dignidad), sin condicionantes económicas, y (c) las personas tengan voz e influyan en la sociedad (poder).

La vulneración de derechos sociales, inequidad social y falta de poder real. A través de múltiples opiniones, los participantes denuncian fallas en el sistema social que resultan en que la gente sea vulnerada en sus derechos, situación frente 
a la cual manifiestan malestar emocional (rabia, impotencia, frustración) y un sentido de urgencia de aportar al cambio. En su visión, existen importantes sectores de la población cuyos derechos sociales están vulnerados, quedando socialmente excluidos y carentes de poder:

Acá también, un trabajador queda igual prácticamente botado, lo echan van $y$ con suerte le pagan indemnización, sería. (E2, 189)

Los jóvenes señalan que hay vidas coartadas porque "todo se compra" y quien no tiene poder de compra queda excluido. Distintas opiniones critican la existencia de un trato indigno de los servicios sociales hacia los pobres:

\section{Terminar con el sistema, terminar, (...) que se colapsan los consultorios, que la gente se muere en los pasillos y eso, digamos, que hay que esperar un mes o dos meses para que alguien lo puedan atender, digamos, en una enfermedad particular. (E5, 84-92)}

Las opiniones de los participantes también remarcan la invisibilización de la pobreza por parte de los medios de comunicación social, la "tristeza" de su realidad y sus problemas sociales asociados (e.g., embarazo adolescente, drogadicción, delincuencia, desprotección en la familia), falta de empoderamiento y dependencia. Por ejemplo, se refieren al conflicto con las tierras del pueblo mapuche, el proceso de erradicación y radicación de viviendas, las escasas oportunidades de educación de los campesinos, los niños en riesgo social y los trabajadores en condiciones laborales desfavorables. La identificación de los jóvenes con la injusticia social y el sufrimiento de las personas afectadas se ilustra en la siguiente cita:

Eh..., no sé si yo seré como muy altruista o, no sé, pero siempre me he preocupado..., siempre me he visto digamos, eh..., al lado de ellos, o sea, siempre he sido parte de ellos y siempre me ha..., llegado mucho a mí mismo, eh... las injusticias y los sufrimientos de ellos. Que ven a sus hijos drogándose y que no puedan hacer nada y que tienen que levantarse a las cinco de la mañana pa' ir a trabajar y vuelven a las nueve de la noche, y..., y esas dos horas que están en su casa, digamos, a su mujer ya no la tocan, a sus hijos no los conocen, no los ven crecer y realmente, personalmente yo, nunca pienso en mí, digamos, pero yo tampoco quiero vivir eso y no me gustaría que mi familia y que toda la gente que quiero viva en eso. $Y$ pienso que realmente toda la gente vive en eso. $(\mathrm{E} 5,24)$

Los jóvenes perciben una desigualdad social contrastante, tanto en el sistema educacional como en el trabajo, la salud, la segregación espacial urbana y las condiciones materiales de los chilenos:

$Y$ hay personas en este país que no tienen que comer y el otro tiene un chorreo de plata. (GF1, 99)

(...) no van a atender [servicios de salud] de un modo a un niño en distintas partes. Eso depende súper harto de la plata. $(\mathrm{E} 6,149)$

Insuficiente respeto por los derechos sociales de los jóvenes. Los participantes reconocen que, aunque puede haber intención de proteger sus derechos sociales y culturales, en la práctica no se respetan los derechos a la educación, salud y trabajo, debido a la desigualdad social que aqueja a la sociedad chilena. En su visión, los derechos sociales de los jóvenes están condicionados por el poder adquisitivo o la situación económica, la apariencia física y la posición social, lo cual limita el desarrollo de sus potencialidades. Como ciudadanos, aspiran a que los derechos y oportunidades estén garantizados por ser partícipes de una sociedad y no por la posición social.

En el mundo del trabajo estos jóvenes perciben que sus derechos son pasados a llevar por los bajos salarios, los obstáculos de ingreso al sistema laboral y la desvalorización de sus capacidades:

Eso es, uno en los trabajos es pasado a llevar porque es un cabro joven. ¿Qué te va a decir un cabro joven? No, no tiene experiencia (...). (E4, 104)

Perciben desconsideración hacia los jóvenes en las oficinas públicas, trabas buro- 
cráticas y la necesidad de pagar por servicios que, en su visión, deberían estar garantizados como derechos.

Barreras a la influencia social: falta de poder. En el ámbito político, los participantes coinciden ampliamente en que, tanto los jóvenes como los demás ciudadanos, no son validados ("no cuentan para las autoridades") y son ignorados a la hora de tomar decisiones. Si bien reconocen que se respeta el derecho de expresión y manifestación pública, ello no va acompañado de un poder ("ser tomado en cuenta"), lo que refleja un sentimiento de baja eficacia política relacionada con una distribución inequitativa del poder en la sociedad. Como ejemplos citan que las reivindicaciones centrales del movimiento estudiantil del 2006 fueron desoídas, que existe represión a los mapuches en sus luchas reivindicativas, desatención a las peticiones de jóvenes ecologistas y una escasa consideración de la demanda de infraestructura para el desarrollo cultural comunal:

Yo creo que existen, pero que no son suficientes es claro, pero de que están, están. Pero el problema, yo creo, más allá de que estén o no estén [los espacios], es que no tienen la validez o el peso que deberían tener, no tienen la eficacia a nivel social o a todo nivel que deberían tener. Yo creo que por ahi va el cuento, porque de existir, existen. Pero los espacios no tienen la eficacia ni nos garantizan que nos van a escuchar o que van a servir para algo posterior o algo más. (GF1, 111)

En la visión de los participantes, los jóvenes requerirían una movilización de gran magnitud para ser visibilizados:

Pero, por ejemplo, con lo que pasó el año pasado [movilización estudiantil en el año 2006] fue necesario mucha revolución como para que de verdad se tomaran en cuenta. $(\mathrm{E} 6,121)$

Los participantes consideran que en la sociedad los jóvenes son percibidos como personas inferiores (e.g., se les asignan tareas de menor importancia), descalificados en su capacidad para formular propuestas, crear y participar, y son etiquetados negativamente (e.g., "carreteros", individualistas, consumistas y arribistas), todo lo cual disminuye su poder de influencia en la sociedad:

De ver a los jóvenes, de repente se nos ve como gente un poquito inferior (...) (E4, 106)

Las respuestas de los participantes también expresan escepticismo y desconfianza frente a los representantes del Gobierno, a quienes atribuyen actitudes autocomplacientes ("hacen como si nos tomaran en cuenta pero es falso"). Refiriéndose a representantes del Gobierno, los participantes describen:

(...) hay otros que son populistas así como que nosotros sí escuchamos a los jóvenes, tomamos su opinión pero, en realidad, es para reafirmar lo que ellos están pensando no más. (GF3, 91)

Para los participantes, los canales de participación existentes han sido diseñados para mantener el control sobre los jóvenes lo cual ha mermado su credibilidad y ha generado desesperanza:

(...) en el día a día, en la gente de mi edad, es que cada vez le importa menos o a lo mejor tienen opiniones, pero sienten que no van a poder hacer nada con esas opiniones. (GF1, 53)

En síntesis, los participantes perciben que las cúpulas políticas no respaldan los proyectos juveniles ("privilegios de 'caudillos' que no representan a los ciudadanos") y que utilizan sus bases juveniles como "mano de obra", imagen y apaciguamiento juvenil.

En forma similar, manifiestan su decepción frente a los canales actuales de participación juvenil, considerándolos "un engaño" en cuanto a la posibilidad de influencia real:

Yo siento que no [hay canales de participación]. Sé que los hay, pero prácticamente la mayoría o solo los que están hechos por jóvenes, todos los demás son mentiras. Siento que la mayoría son para calmarnos, para apaciguarnos, para hacernos sentir que estamos haciendo algo no más. Pero de allí, nos hacen preguntas, las anotan y luego se van y las notas las botan a la basura. O sea, hay canales, pero son de mentira. Uno 
no puede ver los resultados en esos canales a través de lo que intervino, para saber que en verdad están funcionando. (GF1, 107)

Otros jóvenes consideran que los canales de participación existentes son de difícil acceso; por ejemplo, en las universidades privadas la participación es muy limitada. Asimismo, perciben que se les otorga baja credibilidad y pocas oportunidades para asumir roles de importancia en las acciones de intervención social.

Limitaciones del sufragio como mecanismo de influencia ciudadana. Abogando por una ciudadanía activa, los estudiantes identifican las limitaciones del sufragio como expresión de ciudadanía. Definen el sufragio como un mecanismo incompleto, porque no garantiza el cambio social y representa un modo discontinuo ("emisión esporádica de la opinión”) y formal de participación ("muchos votan por deber y solamente una minoría busca el cambio").

La aspiración de ciudadanía de los participantes va más allá del ejercicio del voto; implica ser agente en la transformación de la sociedad a través de la participación y acción social, la responsabilidad social en la vida cotidiana, el sentido de pertenencia, la información y la deliberación por diferentes medios. Desde esa perspectiva, el acto de votar no tiene sentido sin participación permanente:

Se puede votar cada vez que hay elecciones, pero si no se hace nada el resto del tiempo, no va a tener ningún sentido. (GF1, 43)

Alternativamente, en opinión de los participantes, se puede no estar inscrito en los registros electorales, por desencanto o falta de identificación con los partidos políticos, y participar de un modo real. Para ellos, el acto de votar no conlleva necesariamente ciudadanía, debido a la manipulación de políticos, por una parte, y a la desinformación y falta de conciencia de los sufragantes, por otra:

(...) que también está relacionado con la pobreza, los políticos, tú te mueres como los políticos hacen campañas en los campamentos (...) y les pagan a la gente para que sean como militantes de ellos żcachai? y les ofrecen (...) Y cuando están en campañas es cuando más aparecen en las contingencias. Entonces, como que se compran a la gente $y$ la gente vota por ellos. Pero la gente no sabe por quién está votando, no tiene ni idea cuál es el proyecto del que se está candidateando. No saben en qué van a ayudar, cuáles son sus ideologías... ¿̇me entendis? $(\mathrm{E} 3,64)$

Aspiraciones de Ciudadanía de los Jóvenes: Valores de Fraternidad, Dignidad, Equidad y Poder Social

Las motivaciones de cambio y las aspiraciones de ciudadanía de los participantes están enraizadas en valores cívicos y sociales vinculados con la fraternidad, la dignidad expresada en el respeto a los derechos sociales, la equidad y el poder social.

La valoración de la persona en el contexto de relaciones fraternas o comunitarias. Unánimemente, los participantes aspiran a una ciudadanía que cultive las relaciones prosociales (consideración, afecto y respeto de sus miembros). Al describir sus creencias y aspiraciones de ciudadanía, los jóvenes relevan las relaciones comunitarias saludables y el sentimiento de pertenencia en distintos ámbitos de la vida en sociedad: familiar, escolar, comunitaria, la convivencia con pueblos originarios, los grupos u organizaciones en los cuales participan y las relaciones con las personas a quienes apoyan, ayudan o defienden:

Me gusta, me gusta estar, vivir en comunidad y todos conocernos. También he ido al guillatún, he participado hartas veces y la forma que se lleva, la comunidad que está igual... eh... no es como antes, antes era todo comunidad, ahora no, pero sí hay lugares así. Y me gusta, me gusta que así sea (...) y que se ayuden entre ellos porque así, así es el pueblo mapuche. (E2, 213-215)

La valoración de los vínculos afectivos 
también se expresa en la aspiración de los participantes: que las personas por las cuales trabajan en sus proyectos sociales desarrollen una vida familiar plena, que en el colegio se provea un ambiente positivo para el desarrollo y el aprendizaje y que se preserve y extienda la vida comunitaria de los pueblos originarios.

Para los jóvenes la pertenencia también se asocia a un sentimiento de ser útiles, que los motive a participar. En las organizaciones donde desarrollan su trabajo los participantes valoran el clima afectivo y los vínculos interpersonales, particularmente aquellos que muestran preocupación y consideración por el bienestar del otro, y un trato justo:

Claro somos amigos y si le pasa algo a alguien hay que llamarlo, si se muere una persona también, siempre están avisando... pasó esto, pasó esto, nació alguien (...) (E2, 77)

Los jóvenes aprecian los vínculos emocionales (afecto, amistad, compartir tiempo, reconocimiento) y los espacios de convivencia en los cuales las personas se conocen, acogen e incluyen. Asimismo, valoran la solidaridad al interior de su grupo, el compromiso y el compartir metas comunes, todo lo cual refuerza su sentido de comunidad e identificación con la organización a la cual pertenecen:

$E m$, no, es que yo creo que es eso, un poco el sentido de familiaridad dentro de los que lo integramos, un poco como familia $y$... y un poco el ideal, también, que compartimos el mismo ideal en el fondo. $(\mathrm{E} 1,17)$

Asimismo, valoran la protección y la seguridad dada por la comunidad que crean con las personas para las cuales trabajan, ilustrando cómo, a través de la colaboración frente a metas compartidas, se desarrolla confianza social:

Y llega un momento en que te acostumbrai y en verdad yo ahora entro a los campamentos, pero a mis campamentos, como si fueran mi casa, y a las 11 de la noche, porque la gente te conoce y te cuida (...) $(\mathrm{E} 3,12)$
Valoración de la dignidad de la persona: validación de los derechos en un contexto de equidad. En más de una respuesta, todos los jóvenes entrevistados abogan por el respeto de un espectro de derechos sociales y culturales que creen insuficientemente respetados, aspiración que frecuentemente conectan con los objetivos de la organización en la cual participan. El respeto a estos derechos refleja su aspiración de una sociedad en equidad. Manifiestan un sentido de urgencia para la creación de oportunidades de desarrollo de las capacidades, competencias y habilidades de todas las personas, sin distinción socioeconómica, garantizando el acceso a los medios para lograr sus proyectos de vida conforme a sus talentos.

Al describir su trabajo como voluntarios en diferentes organizaciones y/o programas de intervención social, los jóvenes revelan su lucha en defensa de los derechos de otros:

(...) trabajamos con poblaciones "callampa" que se le pueden denominar, donde viven los niños en riesgo social... Generalmente nos llegan niños como de 4 años hasta los 14-15 años, eh, bueno la forma de trabajo es educación popular. Nosotros, lo que consiste nuestro trabajo como monitor de colonias urbanas es mas que todo poder entregarle valores a los niños y traspasar buenas experiencias para ellos, para que se puedan dar cuenta que el mundo no es solamente lo que ellos ven en su población, que el mundo no es solamente drogadicción, que no es solamente alcoholismo, que no es solamente violencia, está toda nuestra intención y toda nuestra energía puesta en poder rescatar a esos niños de la... $(\mathrm{E} 4,2)$

Además del derecho a salud y vivienda, los jóvenes aspiran a que se materialice también el derecho a la educación:

Donde más se ve, por lo menos donde yo más lo veo, es en el ámbito de la educación, que se entregue una educación de calidad para todos (...) (GF3, 175) 
Finalmente, estos jóvenes aprecian el acceso a espacios públicos, como parques y centros culturales, para realizar actividades, el derecho a vivir en un medioambiente equilibrado, y a tener sus tierras para cultivo en el caso de las etnias originarias.

En síntesis, los participantes aspiran a que las personas conozcan sus derechos y deberes, así como también se gesten las condiciones sociales para ejercerlos plenamente:

\begin{abstract}
Para mí un ciudadano es un sujeto que conoce sus derechos y también sus deberes, pero también necesita de condiciones sociales para que pueda ejercer esos derechos y cumplir esos deberes. (GF3, 64) Básicamente, derecho a voto, derecho a educación, eh, derecho a salud, derecho a tener entes reguladores sobre la gestión del Estado o de quienes se preocupan de dónde esté insertado, derecho a vivienda, derecho a un ámbito social ameno, háblese de estética ciudadana, arquitectónica si se quiere decir, espacios públicos, eso. (GF3, 66)
\end{abstract}

La valoración de la distribución equitativa de poder: creencia en una ciudadanía activa y empoderada. Tanto a través de sus opiniones como del testimonio de su accionar, la totalidad de los participantes aspira a una sociedad profundamente democrática. Estos jóvenes creen en una ciudadanía activa que se materializa en los espacios de deliberación y manifestación de las discrepancias. Como ciudadanos, ellos aspiran a tener "voz real" en la toma de decisiones sobre asuntos que les atañen, tanto en sus comunidades como en niveles más amplios de la sociedad. Por lo anterior, consideran insuficiente la instancia del sufragio y la democracia representativa del modelo político vigente, pues perciben que otros deciden por ellos en asuntos de interés y relevancia. Los jóvenes aspiran a que sus opiniones sean tomadas en serio, a influir en la sociedad y a responsabilizarse por estar más informados. Para ello, buscan formas nuevas de organización social, basadas en la horizontalidad de las relaciones y en el establecimiento de puentes adecuados entre las personas y sus representantes:
(...) de hecho, igual el centro de alumnos fue nuestro primer hijo, porque dijimos " $y$ a, mira estas formas de funcionar que tienen ciertas instituciones o ciertas agrupaciones", no funcionan, segregan, o al final alguien se toma el poder, un grupito que empiezan a hacer lo que quiere, o en los partidos políticos se puede ver que empiezan a hacer lo que quieren $y$ entonces nosotros dijimos "¿Cómo lo hacemos?". Empezamos a averiguar formas sociales, empezamos a buscar, por lo menos por mi parte, y también empezamos como a estudiar hartos sociólogos, como somos como bien críticos, empezamos a ver formas nuevas. (GF3, 41)

Según la definición de los participantes, la ciudadanía es activa y comprende una actitud de responsabilidad social, que implica informarse, comprometerse con las necesidades y solución de los problemas de la sociedad, de acuerdo a capacidades y necesidades de cada uno. Respecto de la capacidad de deliberación, algunos jóvenes definen como ciudadanos a personas conscientes de la sociedad y sus mecanismos de funcionamiento e informadas de la realidad local y nacional, más allá de su entorno inmediato.

En el marco de la responsabilidad, los jóvenes valoran una actitud proactiva hacia el candidato político por quien se vota:

(...) por ejemplo, una democracia implica ciudadanos activos, fiscalizadores del accionar de sus representantes, que estén preocupados de las cosas que estén pasando, para que no nos pasen gato por liebre, como siempre nos pasa. (GF2, 46)

Adicionalmente, valoran un compromiso activo con los procesos políticos:

Ciudadanos que estén implicados en los procesos, porque, en última instancia, son todos procesos que les atañen. Ser ciudadano es en todo nivel de cosas, por lo que creo que no se puede ser ciudadano para algunas cosas y para otras no. Uno tiene que ser ciudadano para todo $y$ estar informado para todo, sino se aprovechan y ahi deja de ser democracia $y$ sigue siendo este acuerdo que es como negociación de intereses entre los agentes de poder. (GF2, 46) 
Para los jóvenes, ser ciudadano implica coherencia entre ideas y prácticas, en circunstancias adversas y favorables, evitando la orientación por conveniencia y actuando de manera comprometida, más allá del discurso verbal. Concordantemente, los jóvenes esperan del ciudadano un sentido de responsabilidad social en todo ámbito de su vida (e.g., ejercicio de una profesión). Más allá de lo formal y legal, esperan que influya en su entorno para formar nuevas visiones o prevenir problemas sociales, que sea activista por el bien común y que tenga espíritu solidario. Los entrevistados perciben continuidad entre su identidad ciudadana y su actuación en distintas facetas de su vida:

Yo creo que uno tiene que aprender a ser responsable socialmente en todos los ámbitos de su vida (...) poder siempre tener ese concepto al lado, pegadito, cachai (...) involucrarte en cosas que le pasa a todas las personas. (E3, 62)

En el ejercicio de la ciudadanía, los participantes destacan la importancia de la participación en deliberaciones sobre el proyecto de sociedad, su aspiración a contribuir con ideas y el uso de recursos territoriales:

Para mí, ser ciudadana es participar de la discusión en cuanto a nuestro futuro, a qué queremos ser. Nosotros somos un conjunto de personas, llámese sociedad (...). Tenemos tanto, tantas capacidades, tantos recursos para hacer algo, cómo lo vamos a hacer (...) (E2, 242)

Sus aspiraciones también incluyen informar y crear conciencia sobre determinadas realidades o problemas sociales:

\begin{abstract}
Abrir como... el espacio a la gente para que puedan como,... ser más abiertos de mente, estar más activos frente... y como con los ojos abiertos de lo que pasa alrededor y hacer muchas actividades para que pase eso. $(\mathrm{E} 6,6)$
\end{abstract}

La propuesta de ciudadanía activa de estos jóvenes también considera otros medios, como la creación de coaliciones y movimientos, el diálogo con amplios sectores de la población ante problemáticas y cambios sociales difíciles, la intervención social local a pequeña escala y las instancias de participación a escala humana, con horizontalidad e igualdad. Los jóvenes construyen espacios como colectivos, centros de alumnos y otras organizaciones en los cuales valoran la opinión y creatividad de cada uno. Estos espacios promueven la deliberación y la colaboración en función de metas consensuadas:

Pero en lo que estoy ahora (...) es como bien especial porque nosotros somos quienes delimitamos los planteamientos políticos, discutimos todo el rato, digamos, porque hay, hay bastante diversidad con, con los cabros que estamos trabajando ahora. $(\mathrm{E} 5,4)$

Los participantes objetan la organización jerárquica, la imposición de objetivos o actividades, tanto al interior de sus organizaciones como de parte de otros en relación a ellos como grupo, comunidad o pueblo, ya que, en su visión, atenta contra sus intereses:

Yo estoy más de acuerdo con lo que es colectivos, asambleas, cosas chicas (...). Pero yo creo que lo que es bueno y que se está dando harto ahora, eh, el tema de la asamblea, la transversalidad, no esa jerarquía de partido, señor presidente (...) (E2, 248)

\section{Discusión}

Este estudio documentó las creencias y aspiraciones de jóvenes ciudadanos chilenos y las motivaciones a la base de sus compromisos cívicos. Los participantes perciben deficiencias del sistema social imperante que les motivan a contribuir al cambio social, fundamentalmente, la vulneración de derechos sociales en algunos grupos de la sociedad, la inequidad social y la falta de poder real de los ciudadanos. Esta visión de los participantes ilustra que la ciudadanía es un concepto relacionado con el debate sobre diferencias de poder, NSE y distribución de recursos (Turner, 1993).

Los participantes adscriben a una ciudadanía basada en una cultura cívica de convivencia social que (a) respete la dignidad de cada persona, mediante la inclusión en las estructuras de oportunidades de desarrollo, y los derechos sociales en el marco de relaciones de justicia social; (b) promueva las relaciones saludables de influencia real o empoderamiento y el compromiso con el bien común a diferentes niveles del siste- 
ma social y (c) valore la pertenencia a grupos en los que se experimente un sentido de comunidad.

Los hallazgos anteriores confirman el rol de los valores en el desarrollo de compromisos cívicos y políticos (Flanagan, 2003); estos afectos constituyen la base de las visiones políticas y puntos de vista frente a asuntos públicos (Flanagan \& Faison, 2001). En el curso del desarrollo, niños y jóvenes construyen una comprensión del orden social (Berman, 1997), de los principios a través de los cuales opera y de la medida en que el sistema funciona para personas como ellos. Las experiencias de socialización en la familia y en la escuela y la membresía en organizaciones y programas comunitarios contribuyen a desarrollar un sentido de pertenencia a la comunidad y de que todas las personas importan en una sociedad. Estas experiencias moldean los valores y proveen a los jóvenes de identidades prosociales con las cuales identificarse (Flanagan, 2003). Los valores que sustentan las concepciones de ciudadanía de los participantes - la igualdad, la dignidad, la pertenencia a un contexto de relaciones sociales de cooperación y solidarias- son concordantes con los valores subyacentes a las aspiraciones de todos los chilenos: "la más fuerte y recurrente de las aspiraciones se refiere al deseo que Chile se transforme en una sociedad más igualitaria" (PNUD, 2003, p. 76).

En el plano político los valores que emergen de las concepciones de ciudadanía de los participantes se acercan más a una concepción de republicanismo cívico y democracia participativa que a liberalismo. También coinciden con la visión de ciudadanía de Janoski y Gran (2002), en términos de una membresía en un Estado-nación con derechos universales traducidos en leyes, con obligaciones y capacidades activas para influir en políticas tendientes a la igualdad. Asimismo, sus creencias relevan el respeto por los derechos sociales.

Los participantes aspiran a que la sociedad conforme una comunidad prosocial (Tyler, 2004), cuyo eje sea la inclusión y la participación a través del ejercicio de los derechos y deberes de la comunidad, respetando la dignidad de cada uno y de todos los ciudadanos. El compromiso asumido por los jóvenes entrevistados es un ejemplo de responsabilidad social que ellos proponen para toda la sociedad.

Los jóvenes participantes demandan su derecho a influir por medio de su voz y opinión y a participar en la toma de decisiones en diferentes niveles del sistema social, tanto en las instituciones con las cuales se relacionan como en las políticas públicas. Parcialmente satisfacen esta aspiración en las organizaciones y colectivos en los cuales participan. Sin embargo, no sucede lo mismo con sus propuestas y demandas orientadas a las instituciones del sistema social. En este nivel, critican la falta de reconocimiento de su experiencia y describen barreras en las estructuras sociopolíticas, cuya capacidad para responder a los ciudadanos jóvenes e involucrarlos en la toma de decisiones es baja.

La situación anterior dificulta el proceso de empoderamiento (Rich, Edelstein, Hallman \& Wandersman, 1995), la participación responsable de los jóvenes y genera una brecha entre los ciudadanos jóvenes y las estructuras políticas e institucionales que, en la opinión de los jóvenes, es casi infranqueable ("tienen que llegar a extremos" para ser escuchados). Estos resultados concuerdan con los hallazgos de encuestas nacionales (INJUV, 2007), en las cuales los jóvenes expresan un bajo nivel de confianza en las instituciones políticas y, más de la mitad de ellos, su insatisfacción con el sistema democrático (INJUV, 2007). Adicionalmente, las percepciones de los jóvenes coinciden con el sentimiento de falta de poder que experimenta la mayoría de los chilenos (PNUD, 2004). Todo lo anterior puede afectar negativamente el desarrollo del capital social, ya que, al no sentirse reconocidos ni políticamente eficaces, los jóvenes privan a la sociedad de su contribución renovadora.

El conocimiento de las aspiraciones y valoraciones de los jóvenes facilita que las instituciones que participan en su educación (familia, escuela, organizaciones comunitarias, medios de comunicación social) puedan intencionar su actuación como sujetos participativos y con voz al interior de la sociedad civil. Los resultados sugieren que es prioritario revisar los mecanismos 
de participación vinculante que ofrece la democracia actual y crear oportunidades para una mayor participación en la toma de decisiones de los ciudadanos jóvenes, tanto en los espacios que los congregan como en instituciones formales.

\section{Referencias}

Bárcena, F. (1997). El oficio de la ciudadanía: introducción a la educación política. Barcelona, España: Paidós.

Berman, S. (1997). Children's social consciousness and the development of social responsibility. New York, NY: State University of New York Press.

Domedel, A. \& Peña y Lillo, M. (2008). El mayo de los pingüinos. Santiago, Chile: Editorial Universidad de Chile.

Erikson, E. H. (1968). Identidad, juventud y crisis. Buenos Aires, Argentina: Paidós.

Flanagan, C. A. (2003). Developmental roots of political engagement. PS: Political Science \& Politics, 36, 257-261. doi:10.1017/S104909650300218X

Flanagan, C. A. \& Faison, N. (2001). Youth civic development: Implications of research for social policy and programs. Social Policy Report, 15(1), 3-14.

Garcés, A. (2010). De organizaciones a colectivos juveniles: panorama de la participación política juvenil. Última Década, 18(32), 61-83. doi:10.4067/ S0718-22362010000100004

Ginwright, S. \& James, T. (2002). From assets to agents of change: Social justice, organizing and youth development. New Directions for Youth Development, 96, 27-46. doi:10.1002/yd.25

Instituto Nacional de la Juventud (2004). Cuarta Encuesta Nacional de Juventud: la integración social de los jóvenes en Chile 1994-2003. Santiago, Chile: Ministerio de Planificación y Cooperación.

Instituto Nacional de la Juventud (2007). Quinta Encuesta Nacional de Juventud. Santiago, Chile: Autor.

Instituto Nacional de la Juventud (2009). Sexta Encuesta Nacional de Juventud: principales resultados. Santiago, Chile: Autor. Extraído de http://www.injuv.gob.cl/injuv2010/encuestas_ juventud

Janoski, T. \& Gran, B. (2002). Political citizenship: Foundation of rights. En E. F. Isin \& B. S. Turner (Eds.), Handbook of citizenship studies (pp. 1352). London, Reino Unido: Sage.

Knight, K. \& Harnish, J. (2006). Contemporary discourses of citizenship. Review of Educational Research, 76, 653-690. doi:10.3102/00346543076 004653
Martínez, M. (1999). Comprensión de la cultura no ciudadana en Chile. En Chile, Ministerio Secretaría General de Gobierno (Ed.), Ciudadanía en Chile: el desafío cultural del nuevo milenio (pp. 9-24). Santiago, Chile: Editor, División de Organizaciones Sociales.

Programa de Naciones Unidas para el Desarrollo (2003). Transformaciones culturales e identidad juvenil en Chile. Santiago, Chile: Autor.

Programa de Naciones Unidas para el Desarrollo (2004). Desarrollo humano en Chile. El poder: ¿para qué y para quien? Santiago, Chile: Autor.

Rich, R. C., Edelstein, M., Hallman, W. K. \& Wandersman, A. H. (1995). Citizen participation and empowerment: The case of local environmental hazards. American Journal of Community Psychology, 23, 657-676. doi:10.1007/ BF02506986

Sandoval, J. \& Hatibovic, F. (2010). Socialización política y juventud: el caso de las trayectorias ciudadanas de los estudiantes universitarios de la región de Valparaíso. Última Década, 18(32), 11-36. doi:10.4067/S0718-22362010000100002

Silva, C. L. \& Martínez, M. L. (2007). Empoderamiento, participación y autoconcepto de persona socialmente comprometida en adolescentes chilenos. Revista Interamericana de Psicología, 41, 129-138.

Silva, E. (2009). Challenging neoliberalism in Latin America. New York, NY: Cambridge University Press.

Strauss, A. \& Corbin, J. (1990). Basics of qualitative research: Grounded theory procedures and techniques. Newbury Park, CA: Sage.

Turner, B. S. (1993) Contemporary problems in the theory of citizenship. En B. S. Turner (Ed.), Citizenship and social theory (pp. 1-18). London, Reino Unido: Sage.

Tyler, F. B. (2004). The role of prosocial communities in youth development. Psykhe, 13(2), 3-15. doi:10.4067/S0718-22282004000200001

Velásquez, E. \& Martínez, L. (2004). Participación y género: desafíos para el desarrollo de una política de juventud en Chile. Psykhe, 13(1), 43-51. doi:10.4067/S0718-22282004000100004

Watts, R. J.\& Flanagan, C. (2007). Pushing the envelope on youth civic engagement: A developmental and liberation psychology perspective. Journal of Community Psychology, 35, 779-792. doi:10.1002/ jcop. 20178

Youniss, J., Bales, S., Christmas-Best, V., Diversi, M., McLaughlin, M. \& Silbereisen, R. (2002). Youth civic engagement in the twenty-first century. Journal of Research on Adolescence, 12, 121-148. doi:10.1111/1532-7795.00027 